\title{
The Clock Proteins, Aging, and Tumorigenesis
}

\author{
R.V. KondRATOV* AND M.P. ANTOCH ${ }^{\dagger}$ \\ *BGES Department, Cleveland State University, Cleveland, Ohio 44115; ${ }^{\dagger}$ Department of Molecular \\ and Cellular Biology, Roswell Park Cancer Institute, Buffalo, New York 14263
}

\begin{abstract}
Many aspects of mammalian physiology and behavior are driven by an intrinsic timekeeping system that has an important role in synchronizing various biological processes within an organism and coordinating them with the environment. It is believed that deregulation of this coordination may cause the development of various pathologies. However, recent studies using mice deficient in individual components of the circadian system clearly demonstrated more complex interaction of the circadian system with various biological processes. The growing amount of evidence suggests that in addition to their roles in the core clock mechanism, some of the components of the molecular oscillator are involved in modulation of such diverse physiological processes as response to genotoxic stress, regulation of the cell cycle, aging, and carcinogenesis. These new data provide a mechanistic link between deregulation of the circadian system and/or some of its core components and the development of various pathologies, suggesting novel strategies for the disease treatment and prevention.
\end{abstract}

\section{INTRODUCTION}

\section{Circadian Rhythms and Human Disease}

The circadian clock is a universal intrinsic timekeeping system, which regulates many vital physiological and biochemical processes. In mammals, it is organized as a hierarchical network of molecular clocks that are operative in all tissues, with the master clock residing in the hypothalamic suprachiasmatic nucleus ( $\mathrm{SCN}$ ). The master clock is synchronized with the environment by daily changes in the light/dark cycles and transmits information regarding its phase to multiple tissue-specific clocks (Schibler and Sassone-Corsi 2002). Precise coordination of numerous tissue-specific clocks with each other and with the environment, as well as their proper functioning at the cellular level, is recognized now as important factors of an organism's well being. In humans, the malfunctioning of the circadian clock apparatus has been linked to various pathological syndromes, including sleep and mood disorders, jet lag, tumorigenesis (for review, see Kondratov et al. 2007; Sahar and Sassone-Corsi 2007), and, more recently, to hypertension and type-2 diabetes (Woon et al. 2007). Detailed phenotypic analysis of mice with targeted disruption or mutations in core clock genes further advanced our current view on the role of circadian proteins in pathophysiology. Thus, the growing amount of evidence suggests that core clock proteins may have distinct noncircadian functions important for maintaining tissue homeostasis under normal and stress conditions, and their malfunction may contribute to disease development (Ko and Takahashi 2006; Kondratov et al. 2007). Here, we review recent data from our and other laboratories that address complex interaction of mammalian circadian system with such fundamental biological processes as aging and tumorigenesis.

\section{CLOCK/BMAL1 Transcriptional Complex as the Major Regulator of Circadian Output and a Modulator of the Response to Genotoxic Stress}

The current view of the molecular circadian machinery underlying daily oscillations in physiology, metabolism, and behavior describes the CLOCK/BMAL1 transcriptional complex as the major regulator of both core clock function and circadian output. CLOCK and BMAL1 are two basic helix-loop-helix (bHLH)-PAS domain transcription factors that in a form of heterodimer activate expression of target genes through the E-box enhancer elements in their promoter regions. Among the genes induced by CLOCK/BMAL1 are those encoding their own repressors, PERIODs (PERs) and CRYPTOCHROMEs (CRYs), the regulators of Bmall transcription (REV-ERB? and ROR?) as well as multiple clock-controlled genes (CCGs). The expression of CCGs is achieved either directly or indirectly through the activity of the E box containing noncircadian transcription factors (Gachon et al. 2006).

Among other vital physiological processes, the circadian system has been implicated in modulation of an organism's response to various genotoxic treatments. The initial observations that an organism's response to chemotherapeutic drugs and radiation varies depending on time of administration justified the use of chronotherapy in cancer patients (Levi 2001). Several successful clinical trials have conclusively demonstrated that the therapeutic index of any given treatment (the ratio of tumor response to treatment to the amount of damage caused to normal tissues) could be significantly improved by proper timing (Hrushesky 1985; Giacchetti 2002; Kobayashi et al. 2002). Recent studies performed on mice with targeted disruption of individual clock genes provide the mechanistic background for these initial observations.

The key role of major circadian proteins in genotoxic stress response was demonstrated by testing the sensitivity of wild-type, Clock mutant, Bmal1 ${ }^{-1-}$ knockout, and Cry $1^{-1-} \mathrm{Cry}^{-1-}$ double-knockout animals to toxicity induced by the chemotherapeutic drug cyclophosphamide. Wild-type mice display a robust daily rhythm in sensitivity to the drug. Importantly, morbidity and mortality associated with treatment were at their highest levels when cyclophosphamide was administered at the times of day corresponding to minimal functional activity of the CLOCK/BMAL1 complex and the lowest at the peak times of its activity. Consistently, animals with 
mutations or targeted disruption of the Clock or Bmall genes that are characterized with constant low levels of CLOCK/BMAL1 transcriptional activity (Clock/Clock and $\mathrm{Bmal1}^{-/-}$) showed high levels of drug sensitivity at all times tested. Moreover, animals with constant high levels of CLOCK/BMAL1 functional activity due to the lack of circadian repressors (Cryptochrome double-knockout animals) were extremely resistant to the treatment (Gorbacheva et al. 2005). These data suggest that drug sensitivity is affected by the functional status of major circadian trans-activation complex, which translates into different gene expression patterns of its direct and indirect targets. Indeed, as shown recently, many drug metabolizing and detoxification enzymes as well as a number of key regulators of cell cycle progression and genotoxic stress responses display circadian patterns of expression at either the mRNA or protein level (Fu et al. 2002; Panda et al. 2002; Storch et al. 2002; Gachon et al. 2006). Moreover, some of them, such as the Weel kinase and c$M y c$ transcription factor, have been identified as direct transcriptional targets of the circadian CLOCK/BMAL1 trans-activation complex (Fu et al. 2002; Matsuo et al. 2003). As expected, the expression pattern of many of these genes is altered in tissues of circadian mutant mice or in cells with modulated expression of circadian proteins (Gauger and Sancar 2005; Miller et al. 2007). It has been proposed that the abnormal response to genotoxic stress due to the changes in the abundance and/or activity of the key regulators of cell cycle and apoptosis underlies a cancer-prone phenotype reported for mice with a mutation in the Per2 gene (Fu et al. 2002).

The differential sensitivity of various circadian mutant mice to drug-induced toxicity provided the first example for contrasting physiological phenotypes displayed by behaviorally arrhythmic animals and suggested a more complex interconnection between the circadian system and physiology. Detailed analysis of animals and cells deficient in individual core clock components further confirmed the complexity and the multilevel character of circadian modulation of stress response.

It was thus shown that a deficiency in BMAL1 (positive component of the circadian transcriptional feedback loop) sometimes results in the up-regulation of CLOCK/ BMAL1 transcriptional targets, suggesting that CLOCK/ BMAL1 may also work as a transcriptional repressor (Kondratov et al. 2006b). Importantly, while serving as a repressor, CLOCK/BMAL1 can interfere with the ability of other transcription factors to activate their targets, thus attenuating a DNA-damage response. Hence, the circadian clock may act as a master switch that under normal conditions permits or restricts activation of various signaling pathways. Consistently, the deficiency of individual clock components will result in deregulation of inducibility of stress response pathways, which may cause the development of various pathologies, including premature aging and tumorigenesis (see below).

In addition to transcription-based regulation, core circadian proteins PERIOD1 and TIMELESS interact with components of the cell cycle checkpoint system such as ATM/Chk2 and ATR/Chk1, respectively, and are necessary for activation of Chk1 and Chk2 by DNA damage (for review, see Kondratov and Antoch 2007). Importantly, radiation-induced ATM activation followed by ATMdependent phosphorylation of CHK2 is impaired in cells with specific suppression of PER1 with small interfering RNA (siRNA). These data suggest that PER1 may act either as a cofactor for the activation of ATM or as an adapter protein for recruiting ATM substrates. In addition, modulation of PER1 expression alters both the steadystate expression levels and IR-dependent induction of several cell cycle checkpoint-related genes (Gery et al. 2006).

Analysis of the expression pattern of clock genes in tumor cells also revealed significant differences. Thus, expression of Perl is down-regulated in colon cancer (Krugluger et al. 2007), breast cancers (Chen et al. 2005) and endometrial carcinoma (Yeh et al. 2005) partly due to gene inactivation by promoter methylation. It has been suggested that down-regulation of Per 1 may benefit the survival of cancer cells, thus promoting carcinogenesis (Chen et al. 2005). Consistent with this, expression of exogenous PER1 dramatically reduces the growth of several tested cancer cell lines, presumably by increasing the rate of apoptosis and inducing cell cycle arrest (Gery et al. 2006). Finally, as was shown recently, intratumoral delivery of another Period gene, Per2, inhibits tumorigenesis in C57BL/6 mice (Hua et al. 2007).

Taken together, these data establish a strong molecular link between the clock proteins, on the one hand, and response to DNA-damaging agents, cell cycle regulation, and carcinogenesis, on the other hand.

\section{Premature Aging in BMAL1-deficient Mice}

Detailed analysis of mice deficient in various components of the molecular clock revealed a variety of pathological changes, which sometimes are specific for each mutation, and the list of these pathologies is constantly growing. One of the most striking phenotypes has been described in BMAL1-deficient animals, which display various metabolic defects (Rudic et al. 2004), severe joint ankylosis (Bunger et al. 2005), and premature aging (Kondratov et al. 2006a). BMAL1 (brain and muscle ARNT-like protein 1, also known as MOP3, ARNT3, or ARNTL1) belongs to the family of bHLH-PAS domain transcription factors. In complex with its partner, CLOCK, it regulates expression of target genes. Targeted disruption of BMAL1 results in an immediate loss of behavioral rhythmicity, suggesting that BMAL1 is a core component of the mammalian circadian clock (Bunger et al. 2000).

In addition to its well-established role in circadian mechanisms, a growing amount of recent data suggests that BMAL1 is also directly involved in regulation of a variety of physiological processes. This evidence came from the detailed phenotypic characterization of $\mathrm{Bmall}^{-/}$ mice performed in several laboratories, implying BMAL1 in the regulation of adipogenesis (Shimba et al. 2005), glucose and fat metabolism (Rudic et al. 2004), blood pressure, and heart rate (Curtis et al. 2007). In addition, both male and female mice are infertile (Kennaway 2005) and develop idiopathic calcification and ossification of joints later in age (Bunger et al. 2005). The molecular mechanisms leading to the development of these patholo- 
gies are still unclear; however, it is believed that observed deregulation in the endocrine system and increased sensitivity to DNA-damaging agents may contribute to overall pathogenesis (Gorbacheva et al. 2005).

Recently, we have demonstrated that $\mathrm{Bmall}^{-/-}$mice have several phenotypic changes, which may be summarized as segmental progeria (Kondratov et al. 2006a). Being born with an expected ratio and indistinguishable from their wild-type littermates, at the age of 16-18 weeks, BMAL1deficient animals start showing signs of growth retardation. As a result, at the age of 35-40 weeks, the knockout animals morphologically resemble aged mice and die when they are $37.0 \pm 12.1$ weeks old (Kondratov et al. 2006a).

The progeria-like phenotype in $\mathrm{Bmall}^{-/-}$mice is accompanied with many known features of normal and premature aging in animals. The most prominent among them are sarcopenia (age-related reduction of a muscle mass due to a decrease in the number of muscle fibers and their diameter), osteoporosis (reduction of a bone mass), kidney and spleen shrinkage, significant reduction of visceral and subcutaneous fat, and changes in blood cell composition (increased amount of neutrophiles and monocytes and decreased amount of lymphocytes). In addition, $\mathrm{Bmall}^{-/-}$ mice develop various forms of cataracts and cornea inflammation and lose elasticity of skin and hair growth ability early in age (Kondratov et al. 2006a).

The molecular mechanisms underlying the early aging phenotype of Bmall $^{-1-}$ mice are still unclear. Most likely, it results from the superposition of deficiencies in multiple pathways that are normally controlled by BMAL1, including the regulation of glucose and fat metabolism and homeostasis (Curtis et al. 2004; Shimba et al. 2005) and the modulation of the genotoxic stress response (Gorbacheva et al. 2005), as well as direct involvement of BMAL1 in the regulation of reactive oxygen and reactive nitrogen species (ROS/RNS) (Kondratov et al. 2006a). Age-related accumulation of oxidative damage in an organism has been long recognized as one of the major causes of aging and age-associated degenerative diseases (Balaban et al. 2005). Consistent with this view, the level of free radicals in several tissues of BMAL1-deficient animals appeared to be significantly higher than in the tissues of the age-matched wild-type littermates. Importantly, such age-dependent accumulation of ROS/RNS occurs in those tissues that demonstrate severe reduction in size (i.e., spleen and kidney). Moreover, suppression of BMAL1 levels by SiRNA in a cell culture results in an increase of ROS concentration, suggesting a direct involvement of this core circadian protein in ROS homeostasis (Kondratov et al. 2006a).

Noteworthy, BMAL1 is highly conserved between species. Thus, the sequences of the zebra fish and human homologs of the protein are $84 \%$ identical, whereas mouse and human proteins are $99 \%$ identical. Consistent with this, BMAL1 has a similar functional role in molecular circadian oscillator in human, mouse, and fly (BellPedersen et al. 2005). A recently reported $15 \%$ reduction in life span in flies with a mutation in the CYCLE protein (Drosophila homolog of the mammalian BMAL1) (Hendricks et al. 2003) suggests that the role of BMAL1 in aging is conserved through evolution as well.

\section{Low Doses of Radiation Provoke Premature Aging in Clock Mutant Mice}

Although animals with a mutation in the BMAL1 transcriptional partner CLOCK (Clock mutant mice) share some of the phenotypic characteristics of Bmall-deficient animals (i.e., high sensitivity to drug-induced genotoxic stress) (Gorbacheva et al. 2005), they normally do not demonstrate any signs of premature aging. On the contrary, some of the phenotypes are completely opposite to those displayed by Bmall knockout animals. Unlike Bmall-deficient mice that show age-dependent loss of muscle and adipose tissue, Clock mutant animals develop obesity relatively early in their life. It is caused by various metabolic deregulations including hyperleptinemia, hyperlipidemia, hyperglycemia, and hypoinsulinemia (Turek et al. 2005).

Another circadian mutant that showed some of the features of the early aging are $P e r 2^{m / m}$ mice (Fu et al. 2002; Lee 2006), although there are some important differences. First, in $P e r 2^{m / m}$ mice, this phenotype is developed only after exposure of animals to a low dose of irradiation. Second, it is not that severe compared to BMAL1 deficiency and is manifested mainly by early hair graying. Finally, it is accompanied by a higher incidence of tumor formation (Fu et al. 2002). The latter finding supports previous epidemiological studies linking the disruption of the circadian system (due to abnormal working schedules and/or exposure to frequent changes in the light/dark regime) to tumorigenesis (Antoch et al. 2005; Levi and Schibler 2007). Because previous studies of Clock mutant mice did not identify either a higher incidence of spontaneous tumor development or premature aging, in order to explore the potential role of the CLOCK protein in these processes, we exposed wild-type and Clock/Clock mice to a low dose of ionizing radiation and monitored them for any changes in their gross appearance for 80 weeks following the treatment.

As we reported recently, both wild-type and Clock/Clock mice showed no differences in their acute responses to $4 \mathrm{~Gy}$ of total body irradiation. However, starting at about 20 weeks after the treatment, Clock mutant mice begin to show signs of higher morbidity manifested by progressive weight loss, kyphosis, development of cataracts and eye inflammation, early hair graying, and alopecia. As a result, the survival rate of Clock/Clock mice is significantly reduced compared to similarly treated wild-type mice, and during their life span, they developed a phenotype reminiscent to premature aging in BMAL1deficient animals (Fig. 1). Detailed necropsy performed on animals of both genotypes suggested that the reduction in body weight in Clock/Clock mice resulted from shrinkage of several major organs (liver, spleen, and kidney) as well as from the loss of the adipose tissue. However, no differences in the incidence of tumors were detected in animals of both genotypes (M. Antoch et al., in prep.). Together, these data conclusively demonstrate that although under normal conditions Clock/Clock mice do not display signs of premature aging, exposure to genotoxic treatment such as ionizing radiation accelerates the aging program without affecting carcinogenesis (M. Antoch et al., in prep.). 
A

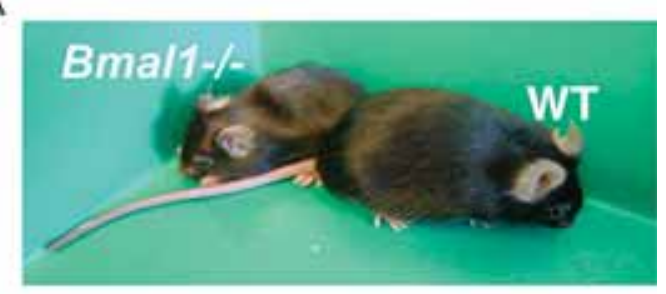

B

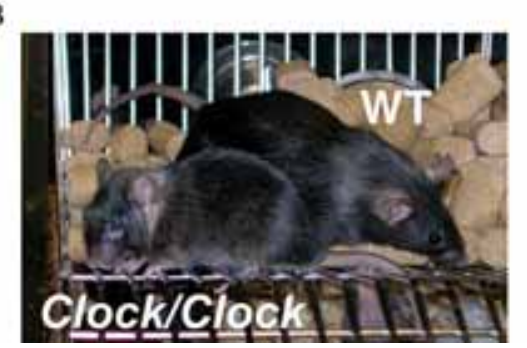

Figure 1. Premature aging in $\mathrm{Bmal}^{-/}$and Clock/Clock mice. $(A)$ Gross appearance of 1-year-old wild-type (WT) and BMAL1deficient mice (Kondratov et al. 2006a). (B) Gross appearance of wild-type (WT) and Clock/Clock mutant mice 80 weeks after exposure to $4 \mathrm{~Gy}$ of total body irradiation (M. Antoch et al., in prep.).

This observation is supported by gene expression experiments demonstrating that the Clock mutation results in down- regulation of several proproliferative genes and upregulation of genes associated with growth arrest and apoptosis (Miller et al. 2007; M. Antoch et al., in prep.) predicting less cancer-prone phenotype. Consistent with this, "clock-less" mice lacking both Cryptochrome genes are indistinguishable from their wild-type littermates in their response to genotoxic treatments (Gauger and Sancar 2005; Gorbacheva et al. 2005). They do not show signs of premature aging or a higher rate of tumorigenesis even after exposure to ionizing radiation (Gauger and Sancar 2005).

The unique progeria-like phenotype described in BMAL1-deficient animals, radiation-induced aging in Clock/Clock mice, or cancer-prone phenotype in Per $2^{\mathrm{m} / \mathrm{m}}$ mutant mice raises an important question on the causal links of these phenotypes with the disruption of the circadian function. The growing list of diverse and sometimes even contrasting phenotypes displayed by behaviorally arrhythmic circadian mutant mice suggests that in addition to their involvement in the molecular clock mechanism, clock proteins may have important roles in maintaining normal tissue homeostasis, and these roles may be distinct from their circadian function (for review, see Kondratov et al. 2007). This becomes particular obvious in comparing the phenotypes of $\mathrm{Bmall}^{-/-}$and Clock/Clock mice.

Indeed, both CLOCK and BMAL1 are required for the formation of an active transcriptional complex that has a central role in circadian-regulated processes. Consistent with this, both Bmall $^{-1-}$ and Clock/Clock mice demonstrate disruption of circadian rhythmicity in behavior and gene expression (Vitaterna et al. 1994; Bunger et al. 2000), as well as some similar changes in metabolic pathways (Rudic et al. 2004). However, in addition to this, they develop a variety of unique phenotypic characteristics. Thus, Clock mutants are obesity-prone, whereas the BMAL1-deficient animals show a "lean" phenotype and defects in adipogenesis (Shimba et al. 2005). In addition, only $\mathrm{Bmal}^{--}$mice normally display premature aging (Kondratov et al. 2006a). These differences may have several explanations. Thus, Clock mutant mice still express functional protein with reduced trans-activation properties and intact transcriptional repressor function (Gekakis et al. 1998; Kondratov et al. 2006b), which makes this model different from the conventional knockout. Because mice with a complete lack of the CLOCK protein have been generated only recently (DeBruyne et al. 2006) and are still not fully characterized, a detailed phenotypic comparison of $\mathrm{Clock}^{-/-}$and $\mathrm{Bmal1}^{-/-}$mice is required to fully address the role of the CLOCK/BMAL1 transcriptional complex and its individual components in maintaining normal tissue homeostasis. In addition, both CLOCK and BMAL1 may interact with unique partners forming the complexes, whose functional activity is independent from the CLOCK/BMAL1 dimer. It has been shown that NPAS2, the closest homolog of CLOCK, which has different tissue specificity of expression, can partially compensate CLOCK deficiency (DeBruyne et al. 2007).

Accelerated aging normally observed in BMAL1-deficient mice and provoked by a low dose of radiation in Per2 and Clock/Clock mutants supports a recently proposed model describing the complex role that the entire circadian system and its core components has under normal and various stress conditions (Kondratov et al. 2007). According to this model, in addition to pathological changes resulting from systemically deregulated circadian function (Fig. 2A), the deficiency of a particular circadian protein may result in the development of a unique set of pathological changes (Fig. 2B). At the same time, because the expression and activities of all components of molecular clock machinery are interrelated, the deficiency of a particular circadian protein will affect the activity of others as well. Therefore, in addition to primary pathology, these animals will have an increased risk for development of secondary pathologies caused by a deficiency of other circadian proteins. In addition, mutations of circadian proteins may affect their interaction with the environment, in which case pathological changes that are not displayed normally will be provoked by environmental stress (Fig. 2C). Accelerated aging of Clock/ Clock mice after exposure to ionizing radiation provides an excellent example for such scenario.

\section{CONCLUDING REMARKS}

The results obtained in our and other laboratories during the past several years clearly demonstrate the importance of the circadian system in mammalian and human physiology. The spectra of pathological changes described in mice with a deficiency of individual components of the molecular clock only partially overlap. Most of the observed pathologies are unique for each individual 
A

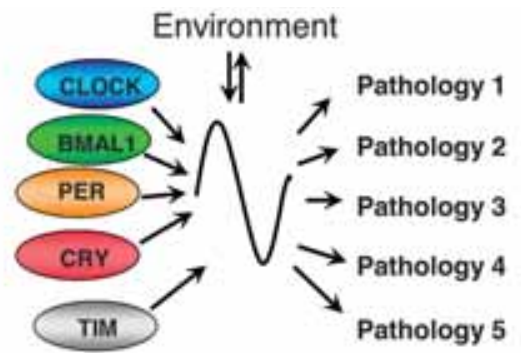

B

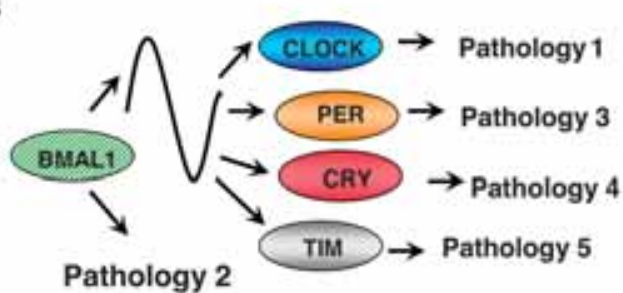

C Environment

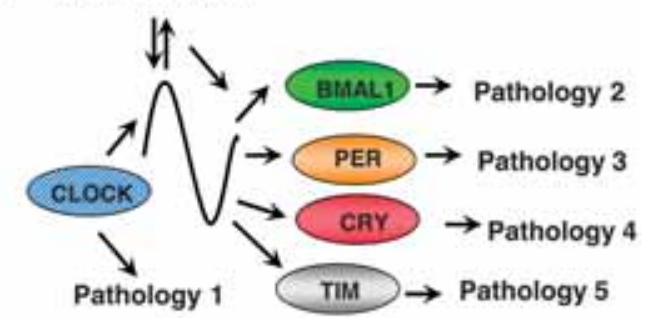

Figure 2. Role of the circadian system, circadian proteins, and organism/environment interactions in development of pathologies. (A) Deficiency in any circadian protein results in deregulation of the circadian system and desynchronization of multiple tissue-specific clocks with each other and with the environment, thus causing various pathological changes. $(B)$ Individual circadian proteins have unique roles in maintaining normal tissue homeostasis that may be relatively independent from their roles in the molecular circadian oscillator. Impaired activity of a particular circadian protein (in this example, BMAL1) will result in a specific primary pathology (Pathology 2). At the same time, since BMAL1 is one of the core components of molecular clock, its deficiency will also affect entire circadian function, leading to a variety of secondary pathologies. (C) Improper interaction of an organism with the environment may affect the expression and/or activity of circadian proteins and induce specific pathologies without directly affecting clock function. (Reprinted, with permission from Kondratov et al. 2007 [ $@$ Elsevier].)

mutant, suggesting that at least some of them develop not as a result of circadian deregulation (because, in this case, they will be identical in all circadian mutants), but as a result of a deficiency in specific noncircadian function of an individual circadian protein.

Most importantly, these results provide molecular mechanisms explaining the increased risk of various diseases in shift workers and frequent time zone travelers. Traditionally, it was proposed that abnormal working schedules result in desynchronization of the tissue-specific molecular clocks within an organism and within an environment, which interferes with an organ's functions through a yet unknown mechanism. However, recent data suggest that exposure to frequent changes in the light/dark cycle may affect the expression of a particular circadian gene, which will cause the changes in the expression and/or activity of other molecular clock components. For example, the functional activity of BMAL1 depends on its expression (regulated by PERs, CRYs, and REV-ERB? proteins) and posttranslational modifications (regulated by CLOCK and CRY proteins). At the same time, BMAL1 is implicated in the control of ROS levels; therefore, any disturbance of the circadian system may have an effect on the activity of BMAL1, which, in turn, through ROS or other mechanisms, will change tissue homeostasis and increase risk of disease.

Taken together, the emerging data of the involvement of circadian proteins in the regulation of many processes in mammalian organisms demonstrate the dichotomy in biological consequences of disruption of the circadian clock with respect to many diseases, including aging and cancer. All of these findings make the circadian proteins perspective targets for the development of novel therapeutic approaches.

\section{ACKNOWLEDGMENTS}

We thank former members of the laboratory, Victoria Gorbacheva and Olena Vykhovanets, for 6 years of productive work that ensured the success of our research program. This work is supported by National Cancer Institute grant CA102522 and NIGMS grant GM075226 (to M.P.A).

\section{REFERENCES}

Antoch M.P., Kondratov R.V., and Takahashi J.S. 2005. Circadian clock genes as modulators of sensitivity to genotoxic stress. Cell Cycle 4: 901.

Balaban R.S., Nemoto S., and Finkel T. 2005. Mitochondria, oxidants, and aging. Cell 120: 483.

Bell-Pedersen D., Cassone V.M., Earnest D.J., Golden S.S., Hardin P.E., Thomas T.L., and Zoran M.J. 2005. Circadian rhythms from multiple oscillators: Lessons from diverse organisms. Nat. Rev. Genet. 6: 544.

Bunger M.K., Walisser J.A., Sullivan R., Manley P.A., Moran S.M., Kalscheur V.L., Colman R.J., and Bradfield C.A. 2005. Progressive arthropathy in mice with a targeted disruption of the Mop3/Bmal-1 locus. Genesis 41: 122.

Bunger M.K., Wilsbacher L.D., Moran S.M., Clendenin C., Radcliffe L.A., Hogenesch J.B., Simon M.C., Takahashi J.S., and Bradfield C.A. 2000. Mop3 is an essential component of the master circadian pacemaker in mammals. Cell 103: 1009.

Chen S.T., Choo K.B., Hou M.F., Yeh K.T., Kuo S.J., and Chang J.G. 2005. Deregulated expression of the PER1, PER2 and PER3 genes in breast cancers. Carcinogenesis 26: 1241.

Curtis A.M., Cheng Y., Kapoor S., Reilly D., Price T.S., and Fitzgerald G.A. 2007. Circadian variation of blood pressure and the vascular response to asynchronous stress. Proc. Natl. Acad. Sci. 104: 3450 .

Curtis A.M., Seo S.B., Westgate E.J., Rudic R.D., Smyth E.M., Chakravarti D., FitzGerald G.A., and McNamara P. 2004. Histone acetyltransferase-dependent chromatin remodeling and the vascular clock. J. Biol. Chem. 279: 7091.

DeBruyne J.P., Weaver D.R., and Reppert S.M. 2007. CLOCK and NPAS2 have overlapping roles in the suprachiasmatic circadian clock. Nat. Neurosci. 10: 543.

DeBruyne J.P., Noton E., Lambert C.M., Maywood E.S., 
Weaver D.R., and Reppert S.M. 2006. A clock shock: Mouse CLOCK is not required for circadian oscillator function. Neuron 50: 465.

Fu L., Pelicano H., Liu J., Huang P., and Lee C. 2002. The circadian gene Period 2 plays an important role in tumor suppression and DNA damage response in vivo. Cell 111: 41.

Gachon F., Olela F.F., Schaad O., Descombes P., and Schibler U. 2006. The circadian PAR-domain basic leucine zipper transcription factors DBP, TEF, and HLF modulate basal and inducible xenobiotic detoxification. Cell Metab. 4: 25.

Gauger M.A. and Sancar A. 2005. Cryptochrome, circadian cycle, cell cycle checkpoints, and cancer. Cancer Res. 65: 6828.

Gekakis N., Staknis D., Nguyen H.B., Davis F.C., Wilsbacher L.D., King D.P., Takahashi J.S., and Weitz C.J. 1998. Role of the CLOCK protein in the mammalian circadian mechanism. Science 280: 1564.

Gery S., Komatsu N., Baldjyan L., Yu A., Koo D., and Koeffler H.P. 2006. The circadian gene per1 plays an important role in cell growth and DNA damage control in human cancer cells. Mol. Cell 22: 375

Giacchetti S. 2002. Chronotherapy of colorectal cancer. Chronobiol. Int. 19: 207.

Gorbacheva V.Y., Kondratov R.V., Zhang R., Cherukuri S., Gudkov A.V., Takahashi J.S., and Antoch M.P. 2005. Circadian sensitivity to the chemotherapeutic agent cyclophosphamide depends on the functional status of the CLOCK/BMAL1 transactivation complex. Proc. Natl. Acad. Sci. 102: 3407.

Hendricks J.C., Lu S., Kume K., Yin J.C., Yang Z., and Sehgal A. 2003. Gender dimorphism in the role of cycle (BMAL1) in rest, rest regulation, and longevity in Drosophila melanogaster. J. Biol. Rhythms 18: 12.

Hrushesky W.J. 1985. Circadian timing of cancer chemotherapy. Science 228: 73 .

Hua H., Wang Y., Wan C., Liu Y., Zhu B., Wang X., Wang Z., and Ding J.M. 2007. Inhibition of tumorigenesis by intratumoral delivery of the circadian gene mPer2 in C57BL/6 mice. Cancer Gene Ther. 14: 815.

Kennaway D.J. 2005. The role of circadian rhythmicity in reproduction. Hum. Reprod. Update 11: 91.

Ko C.H. and Takahashi J.S. 2006. Molecular components of the mammalian circadian clock. Hum. Mol. Genet. (spec. no. 2) 15: R271.

Kobayashi M., Wood P.A., and Hrushesky W.J. 2002. Circadian chemotherapy for gynecological and genitourinary cancers. Chronobiol. Int. 19: 237.

Kondratov R.V. and Antoch M.P. 2007. Circadian proteins in the regulation of cell cycle and genotoxic stress responses. Trends Cell Biol. 17: 311 .

Kondratov R.V., Gorbacheva V.Y., and Antoch M.P. 2007. The role of mammalian circadian proteins in normal physiology and genotoxic stress responses. Curr. Top. Dev. Biol. 78: 173.

Kondratov R.V., Kondratova A.A., Gorbacheva V.Y., Vykhovanets O.V., and Antoch M.P. 2006a. Early aging and age-related pathologies in mice deficient in BMAL1, the core component of the circadian clock. Genes Dev. 20: 1868.

Kondratov R.V., Shamanna R.K., Kondratova A.A., Gorbacheva V.Y., and Antoch M.P. 2006b. Dual role of the CLOCK/BMAL1 circadian complex in transcriptional regula- tion. FASEB J. 20: 530.

Krugluger W., Brandstaetter A., Kallay E., Schueller J., Krexner E., Kriwanek S., Bonner E., and Cross H.S. 2007. Regulation of genes of the circadian clock in human colon cancer: Reduced period-1 and dihydropyrimidine dehydrogenase transcription correlates in high-grade tumors. Cancer Res. 67: 7917.

Lee C.C. 2006. Tumor suppression by the mammalian Period genes. Cancer Causes Control 17: 525.

Levi F. 2001. Circadian chronotherapy for human cancers. Lancet Oncol. 2: 307.

Levi F. and Schibler U. 2007. Circadian rhythms: Mechanisms and therapeutic implications. Annu. Rev. Pharmacol. Toxicol. 47: 593.

Matsuo T., Yamaguchi S., Mitsui S., Emi A., Shimoda F., and Okamura H. 2003. Control mechanism of the circadian clock for timing of cell division in vivo. Science 302: 255.

Miller B.H., McDearmon E.L., Panda S., Hayes K.R., Zhang J., Andrews J.L., Antoch M.P., Walker J.R., Esser K.A., Hogenesch J.B., and Takahashi J.S. 2007. Circadian and CLOCK-controlled regulation of the mouse transcriptome and cell proliferation Proc. Natl. Acad. Sci. 104: 3342.

Panda S., Antoch M.P., Miller B.H., Su A.I., Schook A.B., Straume M., Schultz P.G., Kay S.A., Takahashi J.S., and Hogenesch J.B. 2002. Coordinated transcription of key pathways in the mouse by circadian clock. Cell 109: 307.

Rudic R.D., McNamara P., Curtis A.M., Boston R.C., Panda S., Hogenesch J.B., and Fitzgerald G.A. 2004. BMAL1 and CLOCK, two essential components of the circadian clock, are involved in glucose homeostasis. PLoS Biol. 2: e377.

Sahar S. and Sassone-Corsi P. 2007. Circadian clock and breast cancer: A molecular link. Cell Cycle 6: 1329.

Schibler U. and Sassone-Corsi P. 2002. A web of circadian pacemakers. Cell 111: 919.

Shimba S., Ishii N., Ohta Y., Ohno T., Watabe Y., Hayashi M., Wada T., Aoyagi T., and Tezuka M. 2005. Brain and muscle Arnt-like protein-1 (BMAL1), a component of the molecular clock, regulates adipogenesis. Proc. Natl. Acad. Sci. 102: 12071.

Storch K.F., Lipan O., Leykin I., Viswanathan N., Davis F.C., Wong W.H., and Weitz C.J. 2002. Extensive and divergent circadian gene expression in liver and heart. Nature 417: 78.

Turek F.W., Joshu C., Kohsaka A., Lin E., Ivanova G., McDearmon E., Laposky A., Losee-Olson S., Easton A., Jensen D.R., Eckel R.H., Takahashi J.S., and Bass J. 2005. Obesity and metabolic syndrome in circadian Clock mutant mice. Science 308: 1043.

Vitaterna M.H., King D.P., Chang A.M., Kornhauser J.M., Lowrey P.L., McDonald J.D., Dove W.F., Pinto L.H., Turek F.W., and Takahashi J.S. 1994. Mutagenesis and mapping of a mouse gene, Clock, essential for circadian behavior. Science 264: 719.

Woon P.Y., Kaisaki P.J., Braganca J., Bihoreau M.T., Levy J.C., Farrall M., and Gauguier D. 2007. Aryl hydrocarbon receptor nuclear translocator-like (BMAL1) is associated with susceptibility to hypertension and type 2 diabetes. Proc. Natl. Acad. Sci. 104: 14412.

Yeh K.T., Yang M.Y., Liu T.C., Chen J.C., Chan W.L., Lin S.F., and Chang J.G. 2005. Abnormal expression of period 1 (PER1) in endometrial carcinoma. J. Pathol. 206: 111. 


\section{$8_{\mathrm{CSH}}^{\infty} \mathrm{CH}$ Cold Spring Harbor Symposia SYMPOSIA}

\section{The Clock Proteins, Aging, and Tumorigenesis}

R. V. Kondratov and M. P. Antoch

Cold Spring Harb Symp Quant Biol 2007 72: 477-482

Access the most recent version at doi:10.1101/sqb.2007.72.050

References This article cites 43 articles, 14 of which can be accessed free at: http://symposium.cshlp.org/content/72/477.full.html\#ref-list-1

\section{License} Email Alerting $\begin{aligned} & \text { Receive free email alerts when new articles cite this article - sign up in the box at the } \\ & \text { Service }\end{aligned}$ top right corner of the article or click here.

To subscribe to Cold Spring Harbor Symposia on Quantitative Biology go to: http://symposium.cshlp.org/subscriptions 